\title{
Changing shapes in the nanoworld
}

\author{
Nicolas Combe (a), Pablo Jensen (a) and Alberto Pimpinelli (b) \\ (a) Département de Physique des Matériaux, UMR CNRS 5586, Université Claude Bernard Lyon-1, 69622 Villeurbanne \\ Cédex, FRANCE; \\ (b) LASMEA, Université Blaise Pascal Clermont-2, Les Cézeaux, 63177 Aubière Cédex, FRANCE
}

\begin{abstract}
What are the mechanisms leading to the shape relaxation of three dimensional crystallites ? Kinetic Monte Carlo simulations of fcc clusters show that the usual theories of equilibration, via atomic surface diffusion driven by curvature, are verified only at high temperatures. Below the roughening temperature, the relaxation is much slower, kinetics being governed by the nucleation of a critical germ on a facet. We show that the energy barrier for this step linearly increases with the size of the crystallite, leading to an exponential dependence of the relaxation time.
\end{abstract}

Imagine a world where marbles would fuse upon contact, just as two water droplets usually do to minimize their surface energy and reach their equilibrium configuration. This peculiar behavior is thought to be usual in the nanoworld, because experiments suggest that objects in the panometer range can change shape in reasonable timest. 2, even if they are solid. This fact is crucial for the production and control of nanostructures, since these are generally obtained in out-of-equilibrium conditions, and are therefore metastable. The rapid shape relaxation of these solid particles (which lack the collective atomic diffusion mechanisms found in the liquid state) is dominated by surface diffusion of their surface atoms. Atomic diffusion is random (brownian) but nevertheless generates a global mass transfer from the high curvature regions (of higher chemical potential, roughly because atoms have less neighbors there) to the low curvature regions. For very small objects, surface diffusion is very efficient as a mass transfer mechanism. However, it has to be pointed out that this whole picture assumes that a continuous description of the objects is valid, thus allowing the definition of a curvature-dependent local chemical potential. This is correct as long as the particle is large enough (to allow defining a chemical potential) and has a disordered or rough surface (in order that the chemical potential is differentiable) as for a liquid droplet. Within these assumptions, Herring, Nichols and Mullins 3 have shown that this mass transfer mechanism leads to an equilibrium time $t_{e q}$ which increases as the fourth power of the object linear size, which helps understanding why macroscopic objects are kinetically allowed to violate thermodynamics with total impunity. It is important to note that this fourth power law is extensively used to predict equilibrium times of to extract diffusion constants from equilibration rates明. In this paper, we address the important question of what happens when the temperature is below the roughening transition 5 , so that the particle is faceted, a common situation for nanostructures. There is no general agreement on a continous approach in this regime, and therefore we use Monte Carlo simulations to show that the equilibration time of a faceted parti- cle does not simply vary as predicted above. Indeed, we show that, although surface diffusion remains the important matter transport channel, the equilibration time is limited by another physical mechanism - facet nucleation. This different physical mechanism leads to a very different size dependence of $t_{e q}$, limiting the validity of the continuous law and explaining why nanobjects might spend much more time in metastable equilibrium than expected.

\section{Physical model}

We use standard Kinetic Monte Carlo simulationst to study the equilibration of unsupported 3D crystallites having a perfect fcc crystalline structure. Since we are only interested in finding generic laws for the size dependence of $t_{e q}$ (which should not depend on the details of atom-atom interaction), we have chosen a very simple energy landscape for atomic motionl. We assume that the potential energy $E_{p}$ of an atom is proportional to its number $i$ of neighbors, and that the kinetic barrier $E_{a c t}$ for diffusion is also proportional to the number of initial neighbors, before the jump, regardless of the final number of neighbors, after the jump : $E_{a c t}=-E_{p}=i * E$ where $E$ sets the energy scale $(E=0.1 \mathrm{eV}$ throughout the paper). Comparing with recent ab-initio calculations 10 for the $\mathrm{Al}(111)$ surface, we note that our one-barrier assumption does give the good order of magnitude of the relative jump frequencies for the different hopping processes of interest here. We also exclude any explicit "EhrlichSchwoebel" barrier ${ }^{11}$ for atoms hopping around corners. Therefore, the probability $p_{i}$ per unit time that an atom with $i$ neighbors moves is $p_{i}=\nu_{0} \exp \left[-i * E / k_{b} T\right]$, where $\nu_{0}=10^{13} s^{-1}$ is the Debye frequency. Thus, our simple kinetic model includes only one parameter, the ratio $E / k_{B} T$ where $k_{B}$ is the Boltzmann constant and $T$ the absolute temperature. We have changed the temperature from $300 K$ to $800 K$, and the crystallite number of atoms from 700 up to 13000 . The initial configuration of the clusters is elongated (same initial aspect ratiol2), 
and we stop the relaxation when the crystallites are close to equilibrium, with an aspect ratio of 1.2 .

\section{Simulation results}

Fig. 11 shows a log-log plot of the relaxation time as a function of the number of atoms in the crystallite. The continuous law predicts a slope of $4 / 3$, which agrees with our simulations only for the highest temperature, $800 \mathrm{~K}$. As the temperature decreases, the slope continuously increases, reaching much higher values than this $4 / 3$. This strong deviation from the continuous law suggests that an altogether physical mechanism limits the mass transfer at low temperature. The constant increase of the exponent is a clue that an exponential dependence of $t_{e q}$ on size and temperature might be present.

To investigate the different behaviors at high and low temperatures, we examine (Fig. 2) the different morphologies of the clusters at $T=700 \mathrm{~K}$ and $T=300 \mathrm{~K}$. At high temperatures, many kinks and steps can be seen, indicating that the continuous approximation for the curvature might be valid. On the contrary, at low temperatures the crystallite is fully faceted, with angular points and edges, making it difficult to define a chemical potential properly. Moreover, the presence of facets makes it impossible to transfer atoms from the cluster tips to its central region by simple atomic diffusion : atoms reaching the facets do not find a trapping site there and eventually get back to kinks or steps in the tip regions. This means that the crystallites can be trapped for long times in these faceted configurations at low temperatures, as can be clearly seen on Fig. 3: the cluster progressive approach of equilibrium is continuous at high temperature and more steplike at low temperature, indicating that at high temperature atoms continuously attach to the central regions, whereas some more discontinuous mechanism operates at low temperatures. A careful examination of low temperature relaxation pictures suggested that the transition from one step (i.e. a fully faceted, relatively stable configuration) to the following (lower) step demands the nucleation of a germ on a large facet. This germ grows and eventually forms a new atomic layer, thus bringing the crystallite closer to equilibrium (see Fig. 3). Therefore, we expect that the limiting process for matter transfer at low temperatures is the formation of a critical nucleus, as suggested from classical nucleation theory 13.

This physical picture can be fartly quantified using the kinetic theory of nucleation 13 . The time needed to build the critical nucleus is given by :

$$
t_{n u c l} \propto \exp \left(\frac{\Delta G^{*}}{k_{B} T}\right)
$$

where $\Delta G^{*}$ is the free energy barrier the system has to cross. We stress that here the situation is more complex than in the gas-liquid transition, where the atoms forming the incipient liquid critical cluster all come from the gas phase, which has a fixed chemical potential. Here, the tip atoms come from different environments with different energies (see the different colors in Fig. 2), making it difficult to calculate an average chemical potential. The key point is however to examine whether $\Delta G^{*}$ depends on the particle size, thus creating an exponential contribution to the size dependence of $t_{e q}$.

We use a classical umbrella sampling technique 14 to compute the crystallite free energy as a function of the number of atoms in the nucleating germ. The umbrella technique consists in adding a bias potential to the hamiltonian of the system to force it to stay in a configuration of interest, even if it is unprobable, as is the case here for nucleation of the germ, Fig. 4 shows that $\Delta G^{*}$ increases for larger crystallites 15 , which implies (Eq. 11) that the nucleation time (and therefore $t_{e q}$ ) depends exponentially on the size of the cluster (provided, of course, that the $\Delta G^{*}$ increase is not logarithmic, see below).

What are the microscopic mechanisms leading to this $\Delta G^{*}$ increase with crystallite size? The free energy of a nucleating germ is given by 13 :

$$
\Delta G=2 \gamma_{\text {line }} \sqrt{\pi q}-q \Delta \mu
$$

where $\mathrm{q}$ is the number of atoms in the germ, $\gamma_{\text {line }}$ the line tension of the germ, and $\Delta \mu$ the chemical potential difference for an atom going from the tip to the facet. We fit the curves of Fig. 4 by Eq. 2 which gives $\gamma_{\text {line }}$ and $\Delta \mu$. We find $\gamma_{\text {line }}=0.129 \pm 0.013 \mathrm{eV} /$ atom, independent of the crystallite size. This value is, as expected, close to the binding energy. To understand the size dependence of $\Delta G^{*}$ (which comes from $\Delta \mu$ ), one can, as a first approximation, treat the tips in a continuous way : assimilating them to half an ellipsoid, we can estimate the tip curvature. This gives a rough measure of the kink and step density on the tips, and therefore of the density of more or less mobile atoms, which can contribute to mass transfer. With this approximation, and taking arbitrarily the atom chemical potential to be zero on the facet, we get for the chemical potential difference for an atom going from the tip to the facet :

$$
\Delta \mu=\gamma_{\text {surface }} \kappa
$$

where $\gamma_{\text {surface }}$ is the average surface tension on the tip and $\kappa$ its curvature. Finally, we obtain the free energy barrier for nucleation :

$$
\Delta G^{*}=\frac{\pi \gamma_{\text {line }}^{2}}{\Delta \mu}=\frac{\pi \gamma_{\text {line }}^{2}}{\gamma_{\text {surface }}} \frac{1}{\kappa}
$$

Figure 5 shows that Eqs. 3 and 4 are in good agreement with our simulations and give coherent values for $\gamma_{\text {surface }}$, close to the binding energy $E=0.1 \mathrm{eV}$ (Eq. 3 gives $0.179 \pm 0.008 \mathrm{eV}$ atom $^{-2}$ and Eq. 4 leads to $0.175 \pm 0.035 \mathrm{eV}$ atom $^{-2}$ ).

\section{Conclusion, Perspectives}

The physical picture of nanocrystallite equilibration is the following : above the roughening temperature, the 
continuous approach works well and leads to the classic fourth power law, the mass transfer being via atomic diffusion from kinks or steps from the high curvature regions to the existing kinks or steps of the low curvature region (which act as traps). Below this temperature however, large facets do appear in the low curvature regions and no kinks or steps are available, preventing the diffusing atoms from sticking there. Therefore, the route to equilibrium has to involve nucleation of new atomic planes, which is much more difficult and needs more time, leading to an exponential increase of $t_{n u c}$ (and therefore $t_{e q}$, which is directly related) as a function of the cluster size. We actually see no reason why this picture would not apply to much larger particles, up to the micrometer range. If defect-free particles of this size could be produced, below the roughening temperature they should show perfectly flat facets which would demand the nucleation of a germ for effective mass transfer, thus generating an exponential-type size dependence, and probably prevept ing any experimental observation of the equilibration 16 ! Many open questions remain : the temperature dependence of $t_{e q}$ has to be understood, a more quantitative theory for $\Delta G^{*}$ has to be worked out, and simulations in other geometries (including also the substrate) would also be of interest.

We acknowledge useful discussions with H. Larralde, J.L. Barrat, L. Bocquet, J.J. Métois and J. Krug.

${ }^{1}$ C. R. Stoldt et al., Phys. Rev. Lett. 81, 2950 (1995); A. Ichimiya, Y. Tanaka and K. Hayashi, Surf. Rev. and Lett. 5, 821 (1998).

${ }^{2}$ P. Jensen, Rev. Mod. Phys. 71, 1695 (1999).

${ }^{3}$ C. Herring, Phys Rev 8287 (1951); F.A. Nichols and W.W. Mullins, J. Appl. Phys., 36, 1826 (1965); W.W. Mullins, Metall. and Mat. Trans. A 26, 1917 (1995).

${ }^{4}$ M. Drechsler et al. Journal de Physique 50, Colloque C8, 223 (1989); H. P. Bonzel and E. E. Latta, Surf. Sci. 76, 275 (1978); G. Jeffers, M. A. Dubson and P. M. Duxbury, J. Appl. Phys. 75, 5016 (1994); R. Thouy, N. Olivi-Tran and R. Jullien, Phys. Rev B 56, 5321 (1997); J. Eggers, Phys Rev Lett. 802634 (1998).

${ }^{5}$ A.-L. Barabási and H. E. Stanley, 1995, Fractal Concepts in Surface Growth (Cambridge University Press); J. Lapujoulade Surf. Sci. Rep. 20191 (1994).

${ }^{6}$ H. Spohn, J. Phys. I France 369 (1993).

${ }^{7}$ A. B. Bortz, M. H. Kalos and J. L. Lebowitz, J. Comp. Phys. 1710 (1975); A. F. Voter, Phys. Rev. B 34, 6819 (1986).

${ }^{8}$ H. Shao, P. C. Weakliem and H. Metiu, Phys. Rev. B 53, 16041 (1996); P. Jensen et al. Eur. Phys. J. B 11, 497 (1999).

9 To accelerate our simulations, only particles with less than 7 neighbors are allowed to move : this approximation is especially justified at low temperatures, since motion of atoms with more than 6 neighbors become very rare (there are always some particles with 6 neighbors which move much faster).

${ }^{10}$ A. Bogicevic, J. Strömquist and B. Lundqvist, Phys. Rev. Lett. 81, 637 (1998).

${ }^{11}$ R. L. Schwoebel, J. Appl. Phys. 40, 614 (1969); R. L. Schwoebel and E. J. Shipsey, J. Appl. Phys. 37, 3682 (1966).

12 The aspect ratio is defined as the maximum of all the possible ratios obtained with the gyration radii along the $x$, $y$ and $z$ axis. Actually, it turns out that the initial aspect ratio is not important since the relaxation rate dramatically slows down as the crystallite approaches equilibrium (see Fig. B), and the equilibration time is dominated by the final steps.

13 See for example, Solids Far from Equilibrium, C. Godrèche (Ed.) Cambridge Univ Press (1992); A. Pimpinelli and J. Villain Physics of Crystal Growth (Cambridge University Press, 1998).

${ }^{14}$ D. Chandler Introduction to Modern Satistical Mechanics (Oxford University Press, 1987).

${ }^{15}$ Unfortunately, the umbrella technique gives the free energy to an additive constant. Moreover, the values obtained for $q_{0}<2$ are unphysical since no germ exists. Therefore to be able to compare our curves, we fit them in Fig. 1 with Eq. 2 (which works very well for $q_{0}>2$ ), and we extrapolate the fit down to $q_{0}=0$ to find the free energy value without germ. The additive constant is then fixed for each curve by choosing $\Delta G(q=0$, any $N) \equiv 0$.

16 J.J. Métois and J.C. Heyraud, J. Crystal Growth 57, 487 (1982).

FIG. 1. Log-log dependence of the relaxation time as a function of the size of the crystallites for different temperatures. The slope of each linear fit is indicated.

FIG. 2. Morphologies of crystallites of 1728 atoms at two different temperatures: (a) partially rough at $700 \mathrm{~K}$ and (b) fully faceted at $300 \mathrm{~K}$. The colour of each atom depends on its number of neighbors.

FIG. 3. Evolution of the total energy of crystallites as a function of the time logarithm for a 1728-atom cluster at two temperatures. The arrows in the low temperature curve indicate the transitions from one faceted configuration to the next. The total energy is defined as the number of atomic bonds times the bond energy $(\mathrm{E}=0.1 \mathrm{eV})$. At the end of each curve, the crystallite has almost reached its equilibrium shape. 
FIG. 4. Cluster free energy during the formation of a nucleation germ on a facet as a function of the number $q$ of atoms in the germ. The curves have been obtained at $400 \mathrm{~K}$, for several cluster sizes which have approximately the same shape, close to equilibrium (their aspect ratio is indicated in the figure). Clearly, the free energy barrier for the nucleation of a critical germ becomes larger as the crystallite size increases. Each solid curve is fitted by Eq. 2, allowing to obtain $\gamma_{\text {line }}$ and $\Delta \mu$.

FIG. 5. Dependence of the nucleation barrier (given by the maximum of the curves in Fig. (4) on the tip curvature $\kappa$ (calculated from the crystallite shape). In the inset, we show the dependence of $\Delta \mu$ (deduced from the fits in Fig. (4) on the curvature $\kappa$. We fit these two curves by Eqs. 4 and 3 respectively, obtaining $\gamma_{\text {surface }}$ (see text). 


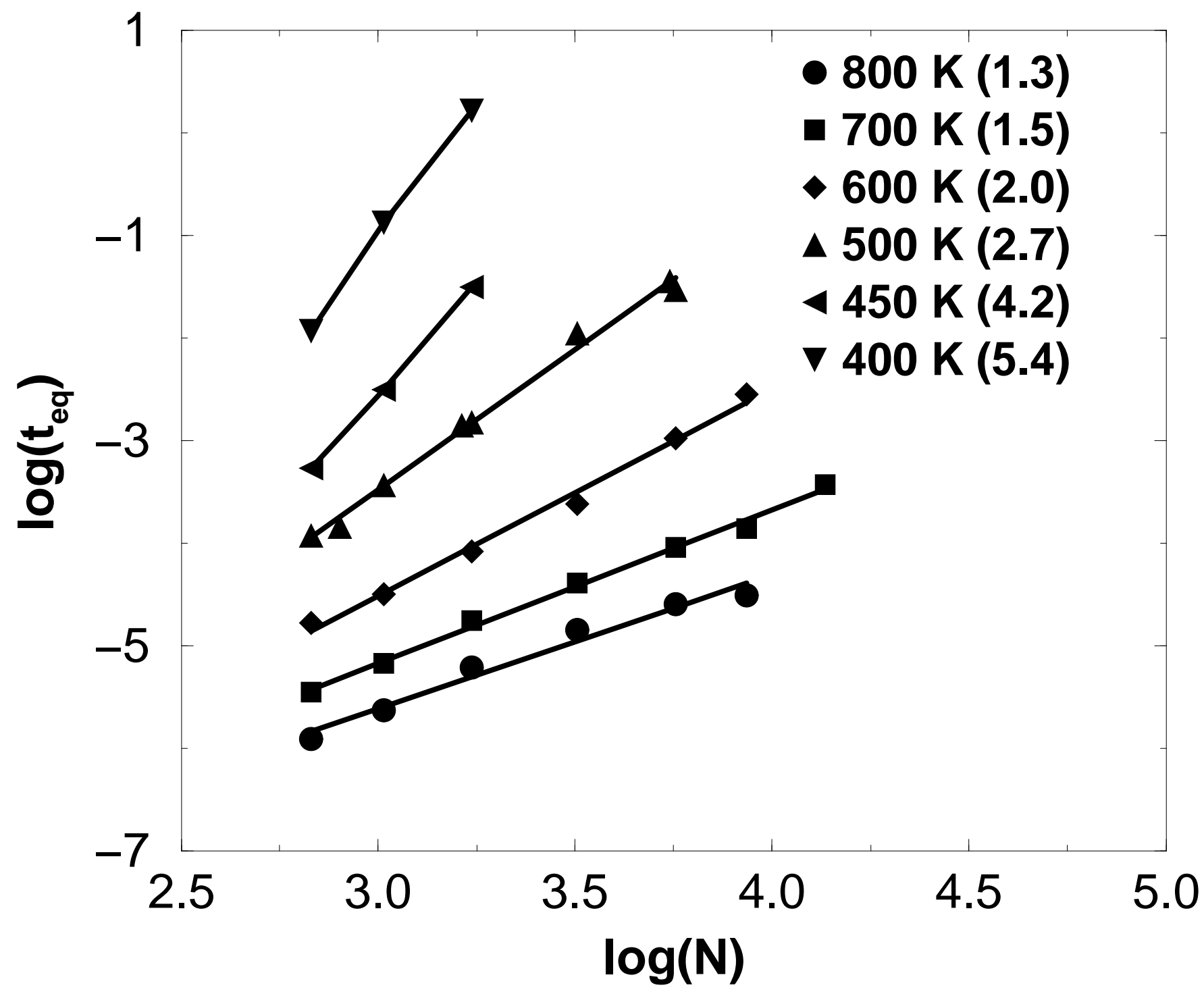




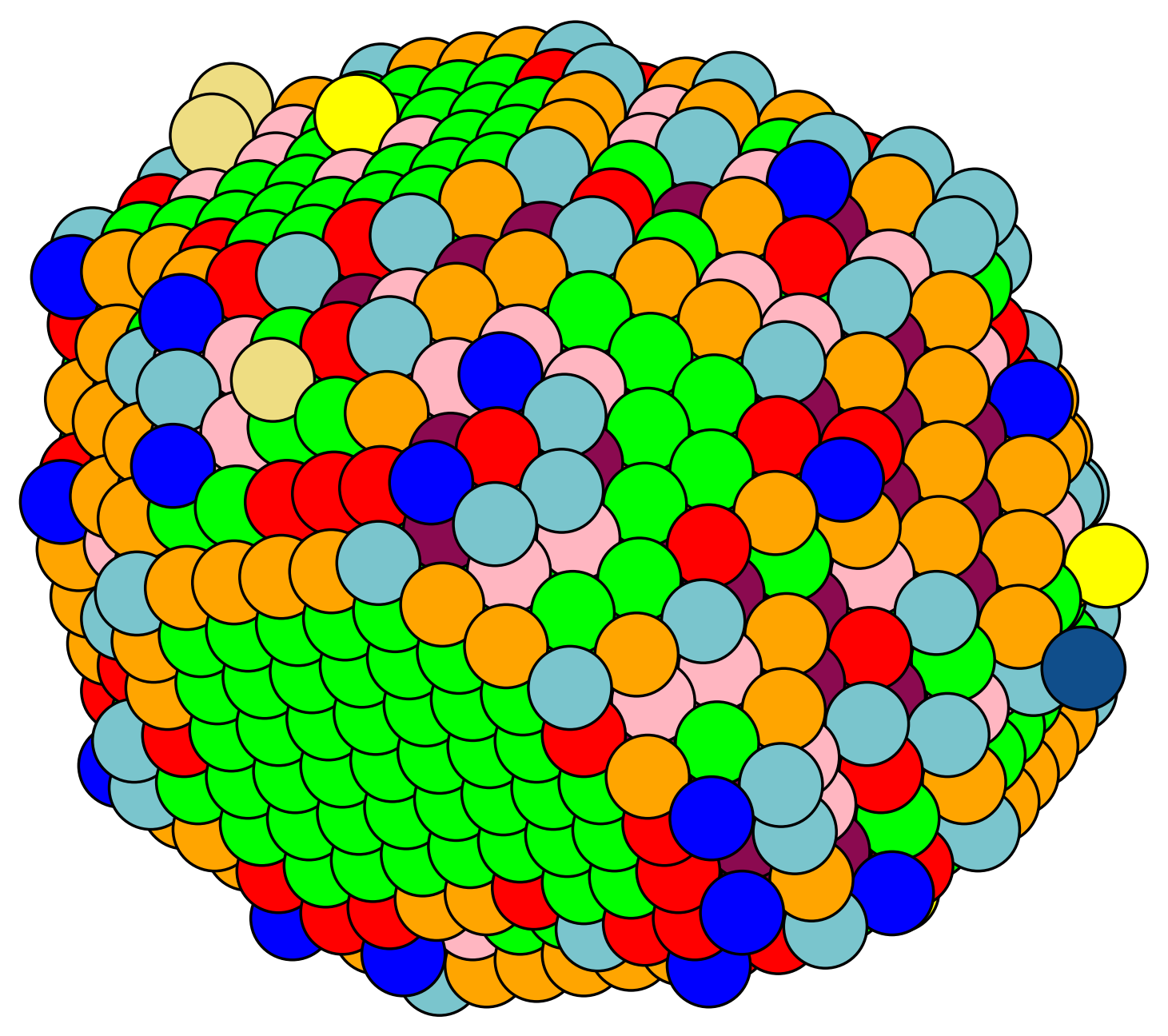




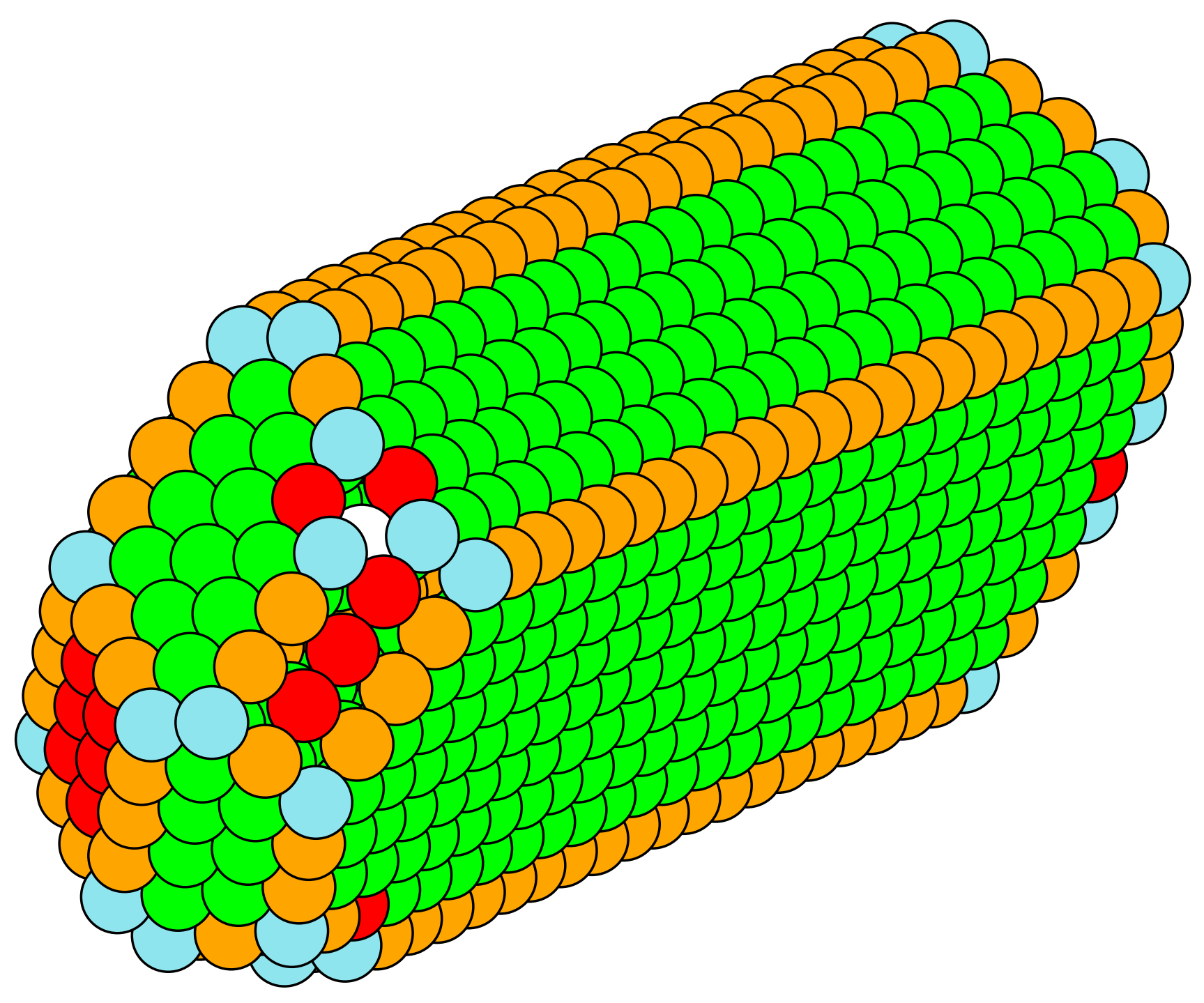




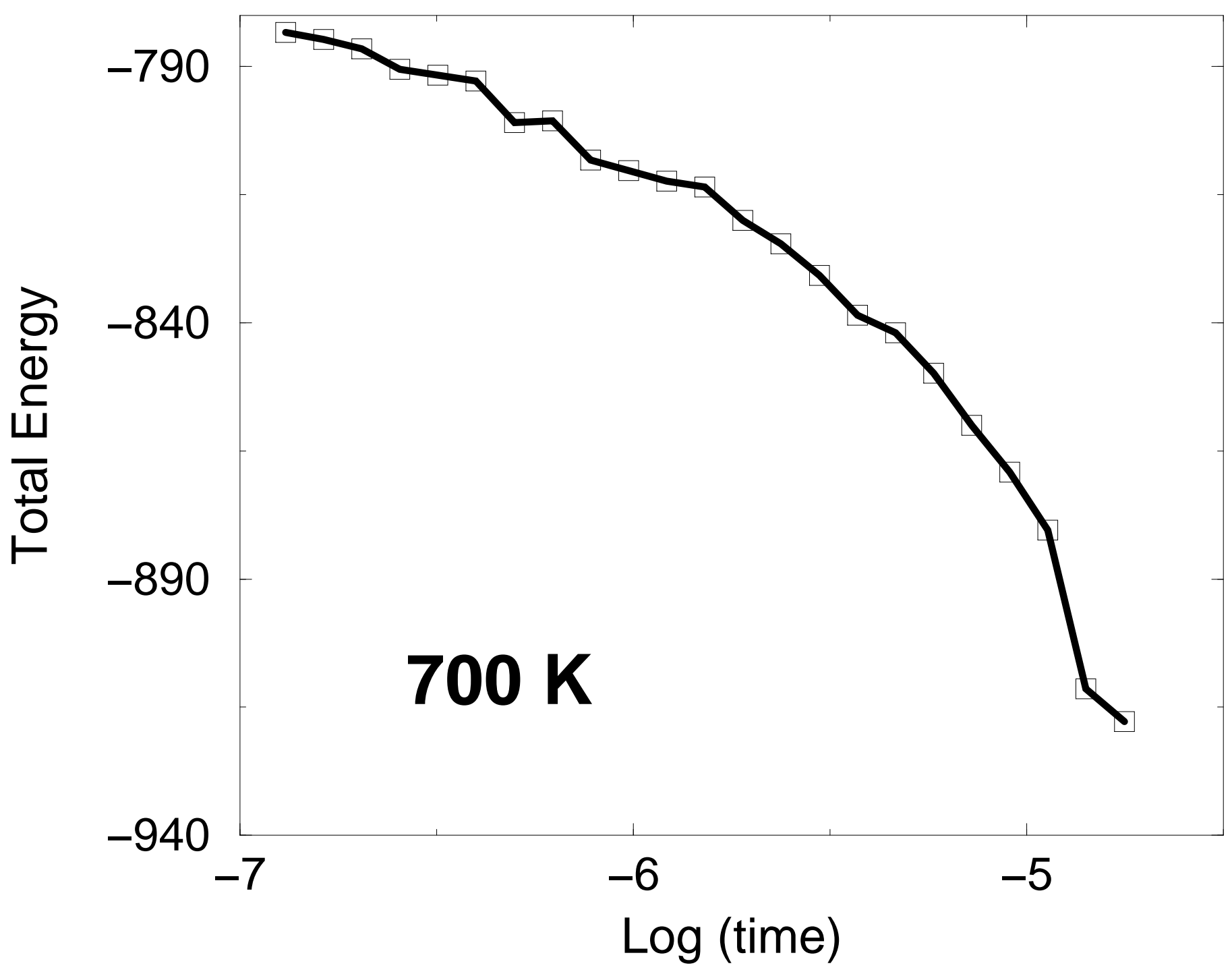




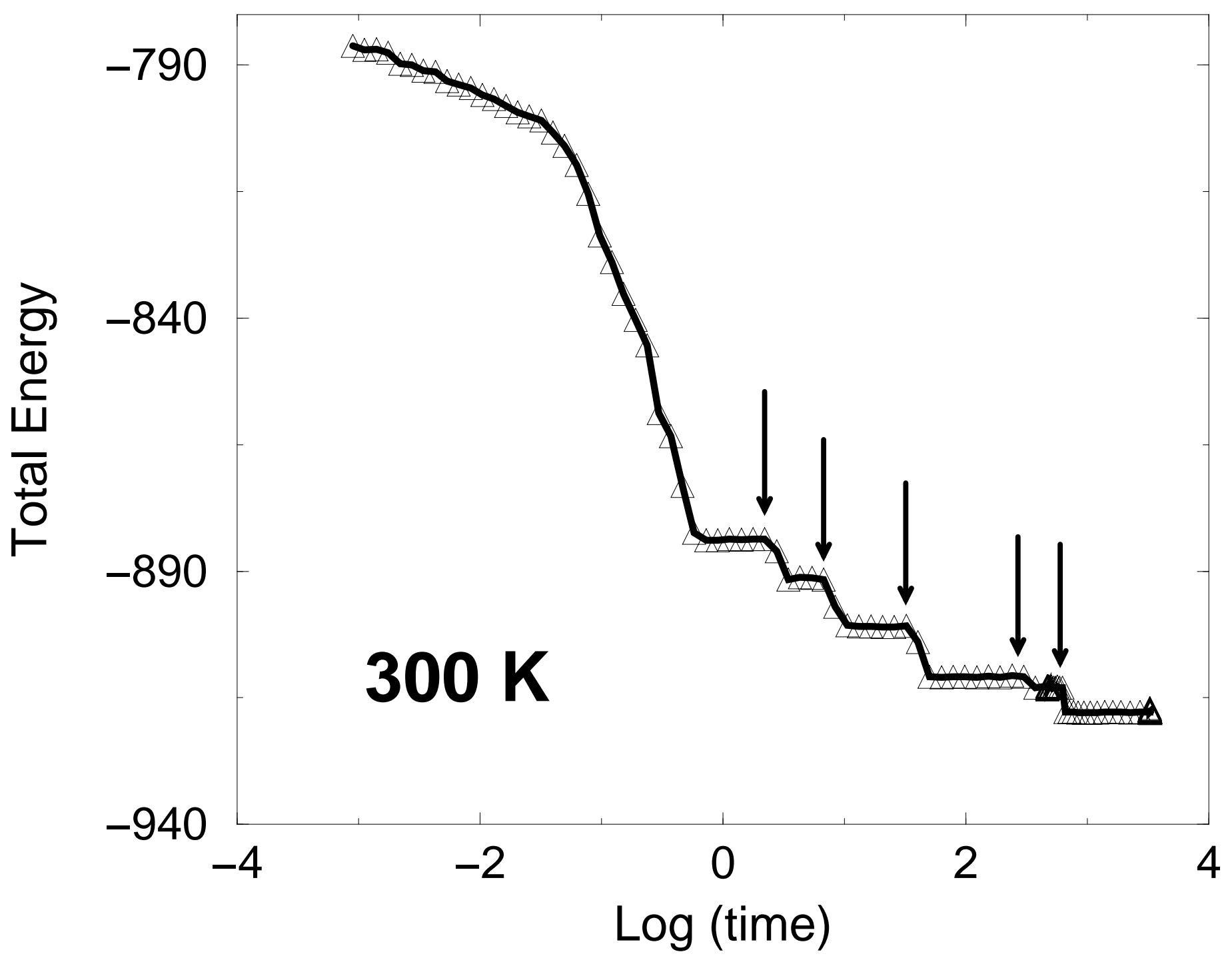




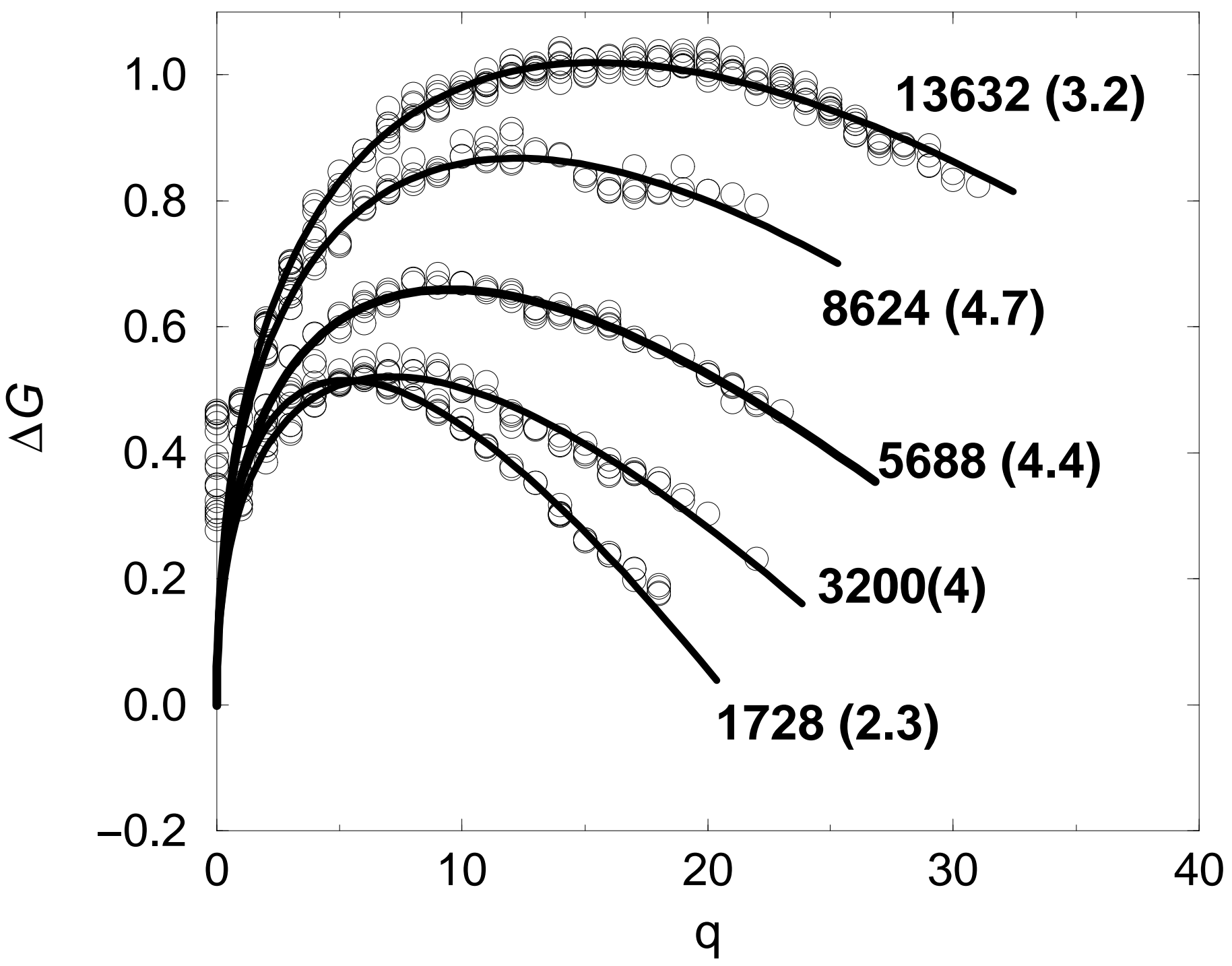




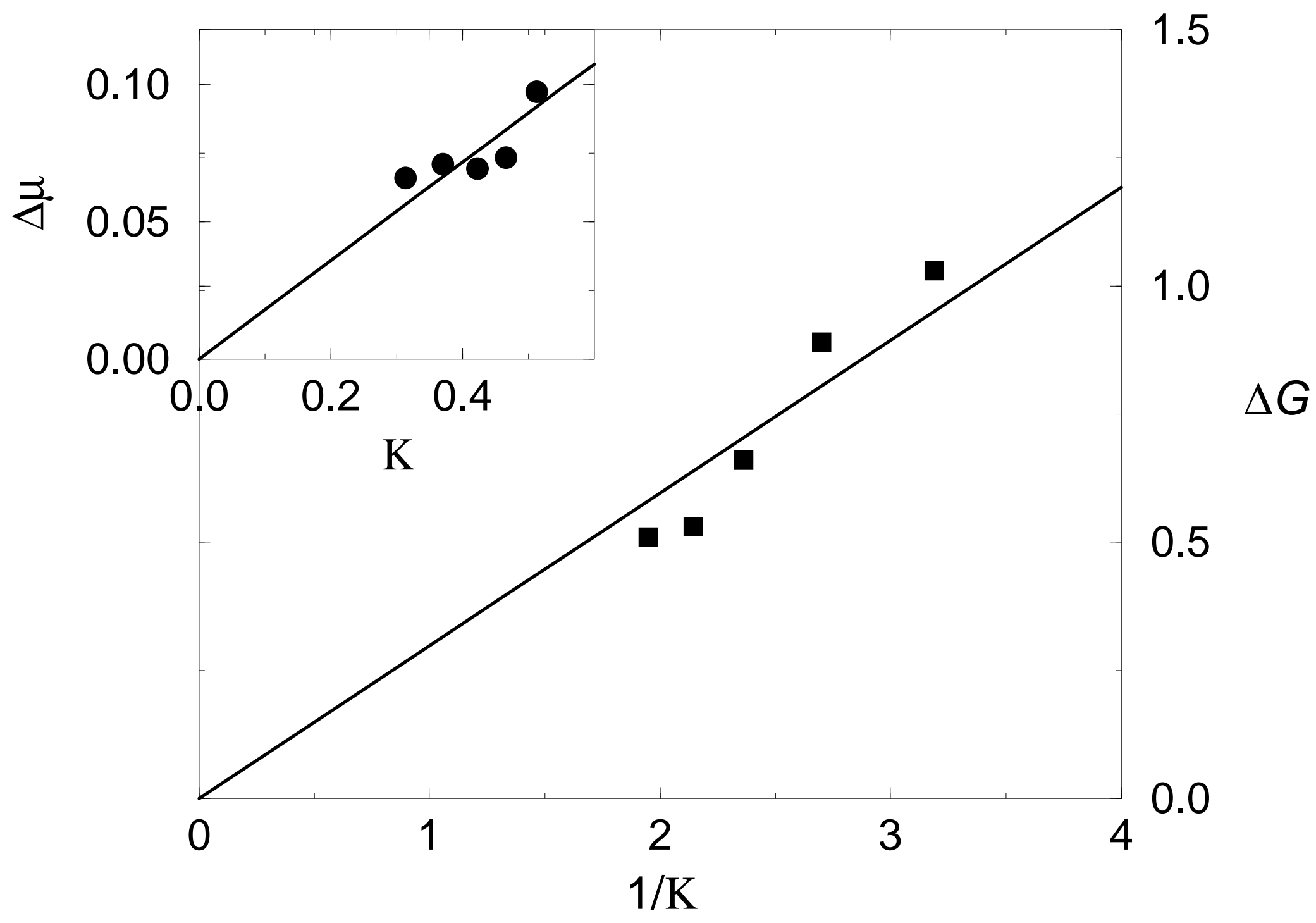

